\title{
Methods of sensitivity theory and inverse modeling for estimation of source parameters and risk/vulnerability areas
}

\author{
Vladimir Penenko ${ }^{1}$ and Alexander Baklanov ${ }^{2}$ \\ ${ }^{1}$ Institute of Computational Mathematics and Mathematical Geophysics, Siberian \\ Division of Russian Academy of Sciences, Lavrentiev av., 6, RUS-630090, \\ Novosibirsk, Russia \\ penenko@ommfao.sscc.ru \\ ${ }^{2}$ Danish Meteorological Institute, Lyngbyvej, 100, DK-2100 Copenhagen, Denmark \\ alb@dmi.dk
}

\begin{abstract}
The source parameters estimation, based on environment pollution monitoring, and assessment of regions with high potential risk and vulnerability from nuclear sites are the two important problems for nuclear emergency preparedness systems and for long-term planning of socio-economical development of territories. For the discussed problems, most of modelers use the common back-trajectory techniques, suitable only for Lagrangian models. This paper discusses another approach for inverse modeling, based on variational principles and adjoint equations, and applicable for Eulerian and Lagrangian models. The presented methodology is based on both direct and inverse modeling techniques. Variational principles combined with decomposition, splitting and optimization techniques are used for construction of numerical algorithms. The novel aspects are the sensitivity theory and inverse modeling for environmental problems which use the solution of the corresponding adjoint problems for the given set of functionals. The methodology proposed provides optimal estimations for objective functionals, which are criterion of the atmospheric quality and informative content of measurements. Some applications of the suggested methods for source parameters and vulnerability zone estimations are discussed for important regions with environmental risk sites.
\end{abstract}

\section{Introduction}

Estimation of source parameters based on environmental pollution monitoring is a very important issue for national emergency response systems. For example, after the Algeciras accident in Spain (30 May 1998) many European monitors measured peaks of air radioactive contamination, but during several days the reason was unknown. Similar situations had happend after the Chernobyl and many others "man-made" catastrophes. Revealing regions with a high potential risk and vulnerability from nuclear or industrial sites is also important in 
the long-term planning of socio-economical development of territories and emergency systems.

A combination of the direct and inverse modelling approaches allows to solve some environmental and nuclear risk problems much more effectively compared with traditional ways based on a direct modelling. In this article we describe some aspects of application and development of the simulation technique proposed in [1-13]. It should be noted that most of researchers, which use for the discussed problems inverse methods, exploit the common back-trajectory techniques, suitable only for Lagrangian models [14-16]. The inverse modeling procedure described in the paper is based on variational principles with splitting and adjoint equations and it is applicable for Eulerian and Lagrangian models $[7,9]$.

Advanced mathematical models of hydrodynamics and models of transport and transformation of pollutants in the gaseous and aerosol states are rather complicated, because they should take into account both natural and anthropogenic factors which affect the processes considered. In one paper it is impossible to give an extensive description of the simulation technique for a whole set of models. Therefore, we present as an example the ideas and main constructional elements of the models of transport and transformation of pollutants. It is assumed that the meteorological characteristics of the atmospheric system are known and prescribed as input information in the transport models.

\section{Formulation of the Problems for Transport and Transformation of Pollutants}

A dual description of the models as shown in $[3,5]$, is used to construct numerical schemes and their algorithms for direct, adjoint, and inverse problems: (1) as differential equations of transport and transformation of multi-species pollutants, and (2) in the variational form with a help of an integral identity.

The basic equation system of the model is written in the following form [4-6]:

$$
(\Lambda \vec{\varphi})_{i} \equiv \frac{\partial \pi \varphi_{i}}{\partial t}+L\left(\pi \varphi_{i}\right)+(H(\vec{\varphi}))_{i}-f_{i}-r_{i}=0, \quad i=\overline{1, n}, \quad n \geq 1,
$$

where $\vec{\varphi}=\left\{\varphi_{i}(\vec{x}, t), i=\overline{1, n}\right\} \in Q\left(D_{t}\right)$ is the vector-function of the state, $\varphi_{i}$ - concentration of the $i$ th pollutant, $n$ - number of different substances, $\vec{f}=\left\{f_{i}(\vec{x}, t), i=\overline{1, n}\right\}$ - source functions, $r_{i}$ - functions which describe uncertainties and errors of the model, $L\left(\pi \varphi_{i}\right)=\operatorname{div}\left[\pi\left(\vec{u} \varphi_{i}-\mu \operatorname{grad} \varphi_{i}\right)\right]$ - advectivediffusive operator, $\vec{u}=\left(u_{1}, u_{2}, u_{3}\right)$ - velocity vector, $\mu=\left\{\mu_{1}, \mu_{2}, \mu_{3}\right\}$ - coefficients of turbulent transfer along the coordinates $\vec{x}=\left\{x_{i}, i=\overline{1,3}\right\}, H(\vec{\varphi})-$ nonlinear matrix operator of an algebraic form, which describes the processes of transformation of pollutants, $\pi$ - pressure function, whose form depends on the chosen coordinate system, $D_{t}=D \times[0, \bar{t}], D$ - domain of variation of the spatial coordinates $\vec{x},[0, \bar{t}]$ - time interval, and $Q\left(D_{t}\right)$ - space of the state functions, which satisfy the conditions at the boundary of the domain $D_{t}$. The transport operator is made antisymmetric using the continuity equation of [3]. 
The initial conditions at $t=0$ and model parameters can be written in the following form:

$$
\vec{\varphi}^{0}=\vec{\varphi}_{a}^{0}+\vec{\xi}_{0}(\vec{x}), \quad \vec{Y}=\vec{Y}_{a}+\vec{\zeta}(\vec{x}, t),
$$

where $\vec{\varphi}_{a}^{0}$ and $\vec{Y}_{a}$ are a-priori estimates of the initial fields $\vec{\varphi}^{0}$ and vector of parameters $\vec{Y} ; \vec{\xi}_{0}(\vec{x}), \vec{\zeta}(\vec{x}, t)$ are the errors of the initial state and parameters. If we suppose that the model and input data are exact, the error terms in (1)-(2) should be omitted. The boundary conditions of the model are consequences of the physical content of the problem under investigation.

If the model implies the presence of errors, the expressions which describe the latter are formally included into the source functions as additional components.

The variational formulation of the model has the form $[3,4]$

$$
I\left(\vec{\varphi}, \vec{\varphi}^{*}, \vec{Y}\right) \equiv \int_{D_{t}}(\Lambda \vec{\varphi}-\vec{f}) \vec{\varphi}^{*} d D d t=0,
$$

where $\vec{\varphi} \in\left(D_{t}\right)$ is the state function, $\vec{\varphi}^{*} \in Q^{*}\left(D_{t}\right)$ - adjoint or co-state function, $Q^{*}\left(D_{t}\right)$ - space of functions adjoint to $Q\left(D_{t}\right), \vec{Y}=\left\{Y_{i}, i=\overline{1, n}\right\} \in R\left(D_{t}\right)$ - vector of parameters of the model, and $R\left(D_{t}\right)$ - region of their admissible values. The integral identity (3) is constructed taking into account the boundary conditions. The integrand in (3) is made symmetric, which automatically (i.e., without additional operations of differentiation and integration) ensures energy balance of functional (3) with the substitution $\vec{\varphi}^{*}=\vec{\varphi}$.

Now let us describe one more essential element of the construction. We mean the data of measurements. In order to include them into the model processing, it is necessary to formulate the functional relationship between the measurements themselves and state functions. Let this relation take the form

$$
\vec{\Psi}_{m}=\vec{M}(\vec{\varphi})+\vec{\gamma}(\vec{x}, t)
$$

where $\vec{\Psi}_{m}$ is the set of observed values; $\vec{M}(\vec{\varphi})$ - set of measurement models; $\vec{\gamma}(\vec{x}, t)$ - errors of these models. The values of $\vec{\Psi}_{m}$ are defined on the set of points $D_{t}^{m} \in D_{t}$.

From point of view of the computational technology, the methods of inverse modeling are more suited to work with global (integral) characteristics of the models and processes than to work with the local ones. This is why we determine the set of such objects in a form of functionals.

For the purposes of monitoring, predicting, controlling, designing, and constructing algorithms of inverse simulation, we introduce a set of functionals

$$
\Phi_{k}(\vec{\varphi})=\int F_{k}(\vec{\varphi}) \chi_{k}(\vec{x}, t) d D d t, \quad k=\overline{1, K}, \quad K \geq 1,
$$

where $F_{k}(\vec{\varphi})$ are the prescribed functions on a set of the state functions, which are differentiable with respect to $\vec{\varphi}, \chi_{k} \geq 0$ - weight functions, and $\chi_{k} d D d t$ 
- corresponding Radon or Dirac measures in $D_{t}$. Using a functional (5) and an appropriate choice of the functions $F_{k}(\vec{\varphi})$ and $\chi_{k}$, we can find generalized estimates of the behavior of the system, ecological restrictions on the quality of the environment, results of observations of various types, purpose control criteria, criteria of the quality of the models, etc. $[5,6]$. For example, measured data (4) can be presented as

$$
\Phi_{0}(\vec{\varphi})=\left(\left(\vec{\Psi}_{m}-\vec{M}(\vec{\varphi})\right)^{T} \chi_{o} S\left(\vec{\Psi}_{m}-\vec{M}(\vec{\varphi})\right)\right)_{D_{t}^{m}},
$$

where the index $T$ denotes the operation of transposition. For source estimation, in addition to the functionals (6), it is necessary to consider a set of functionals in the form (5). Each of them describes an individual measurement.

Variational formulation (3) is used for the construction of the discrete approximations of the model. For these purposes, a grid $D_{t}^{h}$ is introduced into the domain $D_{t}$, and discrete analogs $Q^{h}\left(D_{t}^{h}\right), Q^{* h}\left(D_{t}^{h}\right), R^{h}\left(D_{t}^{h}\right)$ of the corresponding functional spaces are defined on it. Then, the integral identity (3) is approximated by its sum analog

$$
I^{h}\left(\vec{\varphi}, \vec{Y}, \vec{\varphi}^{*}\right)=0, \quad \vec{\varphi} \in Q^{h}\left(D_{t}^{h}\right), \quad \vec{\varphi}^{*} \in Q^{* h}\left(D_{t}^{h}\right), \quad \vec{Y} \in R^{h}\left(D_{t}^{h}\right) .
$$

The superscript $h$ denotes a discrete analog of the corresponding object. Numerical schemes for model (1) are obtained from the stationarity conditions of the functional $I^{h}\left(\vec{\varphi}, \vec{Y}, \vec{\varphi}^{*}\right)$ with respect to arbitrary and independent variations at the grid nodes $D_{t}^{h}$ of the grid functions $\vec{\varphi}^{*} \in Q^{* h}\left(D_{t}^{h}\right)$ for direct problems and $\vec{\varphi} \in Q^{h}\left(D_{t}^{h}\right)$ for adjoint problems [3].

\section{The basic algorithm of inverse modeling and sensitivity studies}

Let us use the ideas of the optimization theory and variational technique for the statement of the inverse problems and construction of methods for their solution. In this case, all approximations are defined by the structure of the quality functional and way of its minimization on the set of values of the state functions, parameters, and errors of the model discrete formulation [5].

The basic functional is formulated so that all the available real data, errors of the numerical model, and input parameters are taken into account:

$$
\begin{aligned}
J(\vec{\varphi})= & \Phi_{k}(\vec{\varphi})+\left(\vec{r}^{T} W_{1} \vec{r}\right)_{D_{t}^{h}}+\left(\left(\vec{\varphi}^{0}-\vec{\varphi}_{a}^{0}\right)^{T} W_{2}\left(\vec{\varphi}^{0}-\vec{\varphi}_{a}^{0}\right)\right)_{D^{h}} \\
& +\left(\left(\vec{Y}-\vec{Y}_{a}\right)^{T} W_{3}\left(\vec{Y}-\vec{Y}_{a}\right)\right)_{R^{h}\left(D_{t}^{h}\right)}+I^{h}\left(\vec{\varphi}, \vec{Y}, \vec{\varphi}^{*}\right) .
\end{aligned}
$$

Here, the first term is given by (5),(6), the second term takes into account the model errors, the third term describes errors in the initial data, the fourth term 
is responsible for the errors of the parameters, and the fifth term is a numerical model of the processes in a variational form, $W_{i},(i=1,2,3$ are weight matrices $)$. The stationarity conditions for the functional (8) gives us the following system of equations:

$$
\begin{aligned}
& \frac{\partial I^{h}\left(\vec{\varphi}, \vec{\varphi}^{*}, \vec{Y}\right)}{\partial \vec{\varphi}^{*}}=B \Lambda_{t} \vec{\varphi}+G^{h}(\vec{\varphi}, \vec{Y})-\vec{f}-\vec{r}=0 \\
& \frac{\partial I^{h}\left(\vec{\varphi}, \vec{\varphi}_{k}^{*}, \vec{Y}\right)}{\partial \vec{\varphi}}+\frac{\partial \Phi_{k}^{h}}{\partial \vec{\varphi}}=\left(B \Lambda_{t}\right)^{T} \vec{\varphi}_{k}^{*}+A^{T}(\vec{\varphi}, \vec{Y}) \vec{\varphi}_{k}^{*}-\vec{\eta}_{k}=0 \\
& \left.\vec{\varphi}_{k}^{*}(\vec{x})\right|_{t=\bar{t}}=0 \\
& \vec{\eta}_{k}(\vec{x}, t)=\operatorname{grad}_{\vec{\varphi}} \Phi_{k}^{h}(\vec{\varphi}) \equiv \frac{\partial \Phi_{k}^{h}(\vec{\varphi})}{\partial \vec{\varphi}} \\
& \vec{\varphi}^{0}=\vec{\varphi}_{a}^{0}+M_{0}^{-1} \vec{\varphi}_{k}^{*}(0), \quad t=0 \\
& \vec{r}(\vec{x}, t)=R^{-1}(\vec{x}, t) \vec{\varphi}_{k}^{*}(\vec{x}, t), \\
& \vec{Y}=\vec{Y}_{a}+M_{1}^{-1} \vec{\zeta}_{k}, \\
& \overrightarrow{\Gamma_{k}}=\frac{\partial}{\partial \vec{Y}} I^{h}\left(\vec{\varphi}, \vec{Y}, \vec{\varphi}_{k}^{*}\right), \\
& \left.A(\vec{\varphi}, \vec{Y}) \vec{\varphi}=\frac{\partial}{\partial \alpha}\left[G^{h}\left(\vec{\varphi}+\alpha \vec{\varphi}^{\prime}, \vec{Y}\right)\right]\right]\left.\right|_{\alpha=0}
\end{aligned}
$$

where $\Lambda_{t}$ is a discrete approximation of the time differential operator, $B$ - diagonal matrix (some diagonal elements of which can be zero), $G(\vec{\varphi}, \vec{Y})$ - nonlinear matrix space operator depending on the state function and parameters, $A^{T}(\vec{\varphi}, \vec{Y})$ - space operator of the adjoint problem, $\vec{\Gamma}_{k}$ - functions of model sensitivity to the variations of parameters, and $\alpha$ - real parameter. The operations of differentiation (9),(10),(12),(16) are performed for all grid components of the state function, adjoint functions, and parameters. For temporal approximation of functional (8), we use the method of weak approximation with fractional steps in time [3]. This is why the equations (9) and (10) are the numerical splitting schemes. The system of equations (9)-(17) is solved with respect to $\vec{r}, \vec{\varphi}^{0}, \vec{Y}$ by the iterative procedures beginning with the initial approximations for the sought functions

$$
\vec{r}^{(0)}=0 ; \quad \vec{\varphi}^{0(0)}=\vec{\varphi}_{a}^{0} ; \quad \vec{Y}^{(0)}=\vec{Y}_{a} .
$$

Three basic elements are necessary for the realization of the method: 1) algorithm for the solution of the direct problem (9),(13)-(15); 2) algorithm for the solution of the adjoint problem (10),(12);3) algorithm for the calculation of the sensitivity functions $\vec{\Gamma}_{k}$ with respect to variations of parameters (16). Then, the main sensitivity relations are constructed $[3,5]$ :

$$
\delta \Phi_{k}(\vec{\varphi})=\left(\vec{\Gamma}_{k}, \delta \vec{Y}\right)=\left.\frac{\partial}{\partial \alpha} I^{h}\left(\vec{\varphi}, \vec{Y}_{a}+\alpha \delta \vec{Y}, \vec{\varphi}_{k}^{*}\right)\right|_{\alpha=0}, \quad k=\overline{0, K} .
$$

where the symbol $\delta$ indicates variations of the corresponding objects, $\delta \vec{Y}=$ $\left\{\delta Y_{i}, i=\overline{1, N}\right\}$ is the vector of variations of the parameters $\vec{Y}$. 
Inverse problems and methods of inverse simulation are formulated from the conditions of minimization of functionals (5) in the space of parameters or from the estimates of sensitivity to variations of these parameters. It includes algorithms for solving problems (9)-(17) and implementation of the feedback from the functionals to the parameters, which are derived from the relations (16) and (19):

$$
\frac{d Y_{\alpha}}{d t}=-\eta_{\alpha} \Gamma_{k \alpha}, \quad \alpha=\overline{1, N_{\alpha}}, \quad N_{\alpha} \leq N,
$$

where $\eta_{\alpha}$ are the coefficients of proportionality, which are found in the course of solving the problem, and $N_{\alpha}$ is the number of refined parameters.

It should be noted that the proposed variational principle, being combined with the splitting technique, allows to derive the hybrid set of Eulerian and Lagrangian transport models $[7,9,8]$. Such types of models complement each other so that their merits increase and demerits decrease in some respect.

In constructing numerical schemes for the transport model, the hydrothermodynamic components of the function of atmosphere state are assumed to be known and can be prescribed by different methods. In particular, they can be calculated from the models of atmospheric dynamics, which are integrated simultaneously with the transport models. For diagnostic studies, evaluation of information quality of observation systems, and implementation of scenarios of ecological prospects, transport models may be used together with models of the informational type, which generate characteristics of atmospheric circulation on the basis of retrospective hydrometeorological information [17]. In this case, for the construction of the directing phase space, we use Reanalysis data [18] for the global and large scales, and DMI-HIRLAM archives [19] for the meso- and regional scales.

The suggested approach enable to solve a broad class of environmental and nuclear risk problems more effectively. This approach includes: (i) development of a methodology to reveal the prerequisites of ecological disasters; (ii) environmental quality modeling; (iii) detection of the potential pollution sources; (iv) evaluation of their intensity; ( $v$ ) estimation of the informative quality of monitoring systems; (vi) calculation of sensitivity and "danger" functions for protected areas; (vii) territorial zoning with respect to the danger of being polluted by known and potential sources of contamination; (viii) interconnections between source and recipient of pollution (direct and feedback relations); (ix) estimation of risk and vulnerability of zones/regions from nuclear and other dangerous sites, and (x) estimation of sensitivity functions for selected areas with respect to risk sites.

The number of applied problems were solved with the help of different modifications of the described methodology. Application of the direct and inverse modeling procedures, including sensitivity investigation, are given in $[7,9,10,13]$. Some specific studies of nuclear risk and vulnerability were presented in $[11,12]$. 


\section{Algorithm for source estimation}

Source term estimation is based on the use of monitoring data and inverse modeling. It is a particular case of the general inverse modeling algorithm $[5,6]$. The source function is considered as one of the parameters in $\vec{Y} \in R\left(D_{t}\right)$. The proposed computational scheme of the algorithm is as follows.

1) Suppose that the source term in (1) can be presented as a function

$$
f=\sum_{\alpha=1}^{\alpha_{N}} Q_{\alpha}(t) \omega_{\alpha}(\vec{x})
$$

where $Q_{\alpha}$ and $\omega_{\alpha}$ are the power and space pattern of the source with the number $\alpha=\overline{1}, \alpha_{N}$.

2) Choose a set of measurements $\Psi_{m}$ and design the form of the functionals $\Phi_{k}\left(\vec{\varphi}, \Psi_{m}\right), k=\overline{1, K}$ for description of the measuremens. Note that the state function have to be included into the functionals for adjoint problems.

3) Calculate the sensitivity functions which can show us if the sought-for source can be observed with a given set of measurements. It means that one has to solve as many adjoint problems as number of measurements. Note, that such problems can be effectively run in parallel computing. Then the obtaned solutions of the adjoint problems should be investigated to find the observability regions where supports of the sensisitivity functions overlap in space and time. One should search for a source namely within these areas. The greater is the number of overlapped supports, the more exact source placement can be isolated.

4) Formulate the sensitivity relations for the chosen set of functionals

$$
\delta \Phi_{k}\left(\vec{\varphi}, \Psi_{m}\right)=\left(\varphi_{k}^{*}(\vec{x}, t), \sum_{\alpha=1}^{\alpha_{N}} \delta Q_{\alpha} \omega_{\alpha}(\vec{x})\right), \quad k=\overline{1, K},
$$

which give us the possibility to define the source power variations. In a linear case, (22) gives the relation for power itself. For more precise identification of the source parameters the general algorithm of inverse modeling should be used.

\section{An example to detect the source location}

A test problem is in the detection of the source location using measurement data. As observational data, the solution of the 3D hemispheric direct problem on pollutant transport from the point source in the Central Europe was taken [10]. The scenario holds a 10-day period (24 March - 02 April 1999). Atmospheric circulation was reconstracted by means of numerical model [17] using Reanalysis data [18] in assimilation mode. The $2 \mathrm{D}$ section of concentration field at the surface level is presented in Fig.1 (left).

Following a criteria of relative significance level of about $10^{-4}$ with respect to the maximum concentration value at 2 April 1999, ten monitoring points, located at the Earth surface, were choosen. The "observations" were provided 
during 1 day. For this monitoring system, the 11 inverse scenarios were created for estimation of the functionals (5) with the linear $F_{k}$ and Dirac measures $[7,9,13]$. The first scenario takes into account the entire monitoring set. In Fig.1 (right), the surface level of the sources' sensitivity function is displayed. The local maximums are obtained near the monitoring sites. As it is seen, there is no direct evidence for detection of the source. The generalized characteristic of the next 10 scenarios is presented in Fig.2 (left). Each of the scenarios gives the sensitivity functions for the functional that takes into account just one observation. The generalized characteristic is the function which describes the overlap of the sensitivity function supports. The value of this function gives the number of overlapped areas. The domains with the great values is preferable to search a source. The value 10 shows the domain where 10 supports overlap. In Fig. 2 (right), which is extraction from Fig.2 (left), this domain is displayed.

Several other examples of source term estimation, based on the direct and inverse local-scale modelling in a case of accidental contamination from an unknown release, and estimation of regional risk and vulnerability from various nuclear and industrial dangerous sites are discussed in $[8,12,13]$.

Thus, the suggested methodology, based on a combination of direct and inverse modeling, extends the area of application of mathematical models. It allows us to find key parameters which are responsible for the processes' behavior. Variational principle provides relations between measured data and models, and gives the construction for a whole set of algorithms to solve environmental problems.

Acknowledgments. V.P.'s work is partly supported by the Grants of the RFBR (01-05-65313 and 00-15-98543) for the Leading Scientific Schools of Russia, Russian Ministry of Industry and Science (0201.06.269/349), and European Commission (ICA2-CT-2000-10024).

\section{References}

[1] Marchuk, G.I.: Mathematical modeling in the environmental problems. Moscow, Nauka (1982) (in Russian)

[2] Marchuk, G. I.: Adjoint equations and analysis of complex systems. Kluber Academic Publication (1995)

[3] Penenko, V.V.: Methods of numerical modeling of the atmospheric processes. Leningrad, Gidrometeoizdat (1981) (in Russian)

[4] Penenko, V.V. and Aloyan, A.E.: Models and methods for environment protection problems. Nauka, Novosibirsk (1985)

[5] Penenko, V.V.: Some Aspects of Mathematical Modeling Using the Models Together with Observational Data. Bull. Nov. Comp. Cent. 4 (1996) 31-52

[6] Penenko, V.V.: Numerical models and methods for the solution of the problems of ecological forecast and design. Survey of the Applied and Industry Mathematics. Vol. 1, 6 (1994) 917-941 

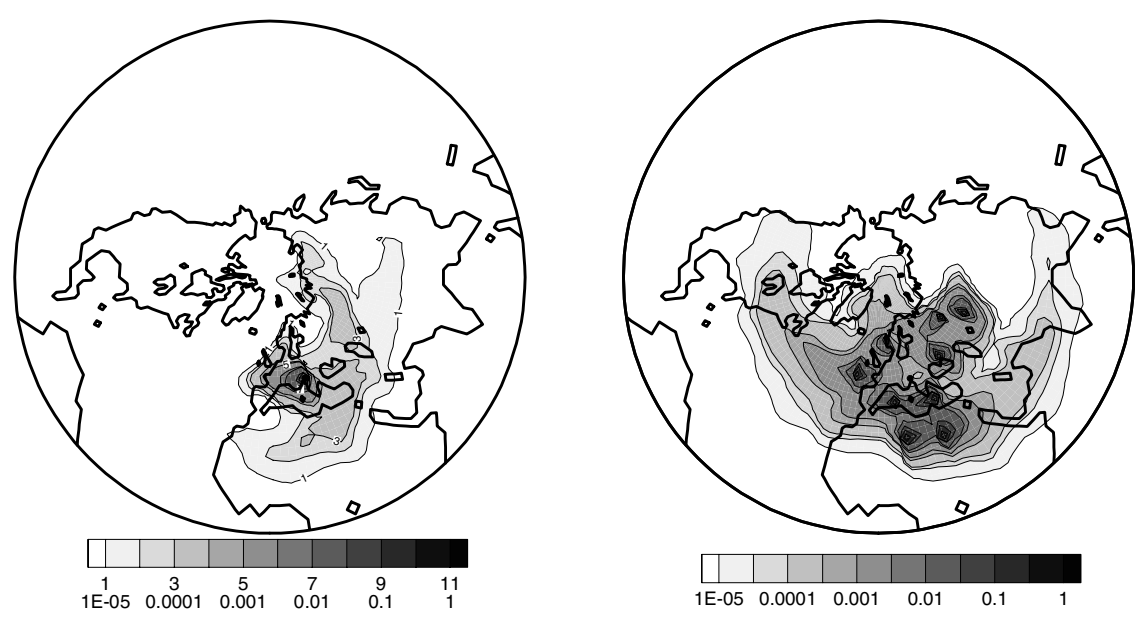

Figure 1: Solution of the direct problem: concentration fields (left), and the sensitivity function based on the inverse simulation for the entire set of observations (right)
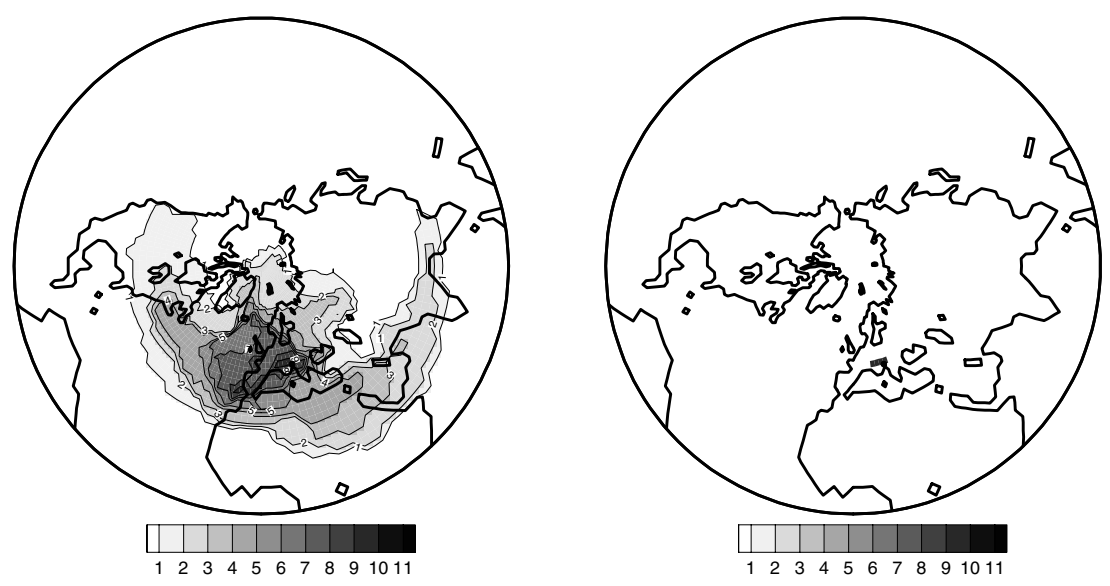

Figure 2: The overlapped supports of the sensitivity functions for 10 individual observations (left) and detected sourse location area (right) 
[7] Penenko, V.V. and Tsvetova, E. A.: Modeling of processes of pollutant transport in direct and inverse problems of climato-ecological monitoring and prediction. Atmos. Ocean. Opt. 12(6) (1999) 462-467

[8] Baklanov, A.: Modelling of the atmospheric radionuclide transport: local to regional scale. In: Numerical Mathematics and Mathematical Modelling, Vol. 2. INM RAS, Moscow, Russia (2000) 244-266

[9] Penenko, V.V. and Tsvetova, E.A.: Some aspects of solving interrelated problems of ecology and climate. Journ. of Applied Mechanics and Technical Physics, 41(5) (2000) 907-914

[10] Penenko, V. V. and Tsvetova, E. A.: Analysis of scales of the anthropogenic impacts in the atmosphere. Atmos. Oceanic Opt., 13(4) (2000) 361-365

[11] Baklanov, A., Mahura, A., Jaffe, D., Thaning, L., Bergman, R. Andres, R.: Atmospheric Transport Patterns and Possible Consequences for the European North after a Nuclear Accident. Journal of Environmental Radioactivity (2001) (accepted)

[12] Rigina, O. and Baklanov, A.: Regional radiation risk and vulnerability assessment by integration of mathematical modelling and GIS-analysis. J. Environment International (2001) (submitted)

[13] Penenko, V.V.: Revealing the areas of high ecological vulnerability: the concept and approach to realization. Atmos. Ocean. Opt. 14 (2001) (in press)

[14] Prahm, L.P., Conradsen, K., Nielsen, L.B.: Regional sourse quantification model for sulphur oxides in Europe. Atmos. Env. 14 (1980) 1027-1054

[15] Persson, C., Rodhe, H., De Geer, L.E.: The Chernobyl accident: A meteorological analysis of how radionuclides reached and were deposited in Sweden. Ambio, 16 (1987) 20-31

[16] Seibert, P.: Inverse dispersion modelling based on trajectory-derived sourse-receptor relationsships. In: Gryning, S.E., Chaumerliac, N.(eds.): Air pollution modeling and its application XII, Plenum, NY (1997) 711713

[17] Penenko, V. V. and Tsvetova, E. A.: Preparation of data for ecological studies with the use of Reanalysis. Atmos. Ocean. Opt., 12(5) (1999) 447449

[18] Kalnay, E., Kanamits, M., Kistler, R. et al.: The NCEP/NCAR 40-year reanalysis project. Bull. Amer. Meteorol. Soc. 77, (1996) 437-471

[19] Sørensen, J.H., Laursen, L., Rasmussen; A.: Use of DMI-HIRLAM for operational dispersion calculations. In: Gryning, S.E., Millan, M.M.(eds.): Air pollution modeling and its application X, Plenum, NY (1994) 373-381 